\title{
AOOT 1974
}

56॰ ANNÉE - N• 668

\section{revue internationale de la croix-rouge}

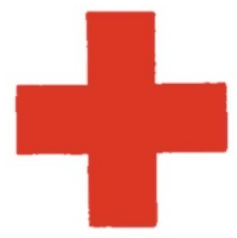

INTER ARMA CARITAS

GENEVE

COMITÉ INTERNATIONAL DE LA CROIX-ROUGE FONDE EN 1863 


\section{COMITÉ INTERNATIONAL DE LA CROIX-ROUGE}

MM. ERIC MARTIN, docteur en médecine, professeur honoraire de l'Université, Genève, président (membre depuis 1973)

JEAN PICTET, docteur en droit, président de la Commission juridique, vice-président (1967)

HARALD HUBER, docteur en droit, juge fédéral, vice-président (1969)

HANS BACHMANN, docteur en droit, directeur des finances de la ville, Winterthour (1958)

MM. MAX PETITPIERRE, docteur en droit, ancien conseiller fédéral (1961)

ADOLPHE GRAEDEL, ancien député au Conseil national. ancien secrétaire général de la Fédération internationale des ouvriers sur métaux (1965)

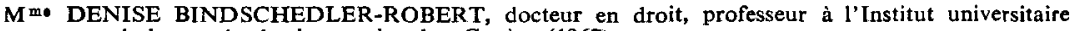
de hautes études internationales, Genève (1967)

MM. MARCEL A. NAVILLE, licencié ès lettres, président du CICR de 1969 à 1973 (1967)

JACQUES F. DE ROUGEMONT, docteur en medecine (1967)

ROGER GALLOPIN, docteur en droit, ancien directeur général du CICR (1967)

WALDEMAR JUCKER, docteur en droit, secrétaire de l'Union syndicale suisse (1967)

VICTOR H. UMBRICHT, docteur en droit, administrateur (1970)

PIERRE MICHELI, ticencié en droit, ancien ambassadeur (1971)

GILBERT ETIENNE, professeur à 1'Institut universitaire de hautes études internationales et à l'Institut d'études du développement, Genève (1973)

ULRICH MIDDENDORP, docteur en médecine, chef de la clinique chirurgicale de 1"Hôpital cantonal, Winterthour (1973)

M"ue MARION ROTHENBACH, diplômée M.S.W. de l'Université de Michigan, maítre-assistant à l'Ecole des sciences sociales et politiques de l'Université, Lausanne (1973)

MM. HANS PETER TSCHUDI, docteur en droit, ancien conseiller féđéral (1973)

HENRY HUGUENIN, directeur de banque (1974)

GOTTFRIED DE SMIT, administrateur (1974)

Membres honoraires: M. JACQUES CHENEVIÈRE, vice-président d'honneur; $\mathrm{M}^{11 \mathrm{e}}$ LUCIE ODIER, vice-présidente d'honneur; MM. GUILLAUME BORDIER, PAUL CARRY,

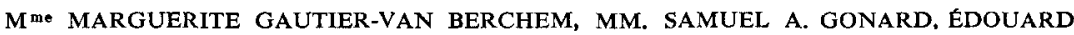
DE HALleR, RODOLFO OLGIATI, PAUL RUEGGER, DIETRICH SCHINDLER, FREDERIC SIORDET, ALFREDO VANNOTTI.

\section{CONSEIL EXÉCUTIF}

M. ROGER GALLOPIN, président

$M$. VICTOR H. UMBRICHT, vice-président

$M^{\text {mo }}$ DENISE BINDSCHEDLER-ROBERT

M. GILBERT ETIENNE

D ULRICH MIDDENDORP

M. JEAN PICTET

M. GOTTFRIED DE SMIT

M. PIERRE MICHELI, membre suppléant 


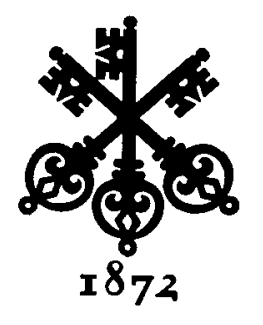

PRÉSENTE SUR LES CINQ CONTINENTS, LA GRANDE BANQUE AUX TROIS CLEFS ÉST À VOTRE DISPOSITION POUR EFFECTUER TOUTES VOS OPÉRATIONS FINANCIĖRES

\section{SOCIÉTÉ DE BANQUE SUISSE}

SCHWEIZERISCHER BANKVEREIN - SOCIETA DI BANCA SVIZZERA

SWISS BANK CORPORATION

SIËGE DE GENĖVE

2, RUE DE LA CONFÉDÉRATION

TÉL. 201111

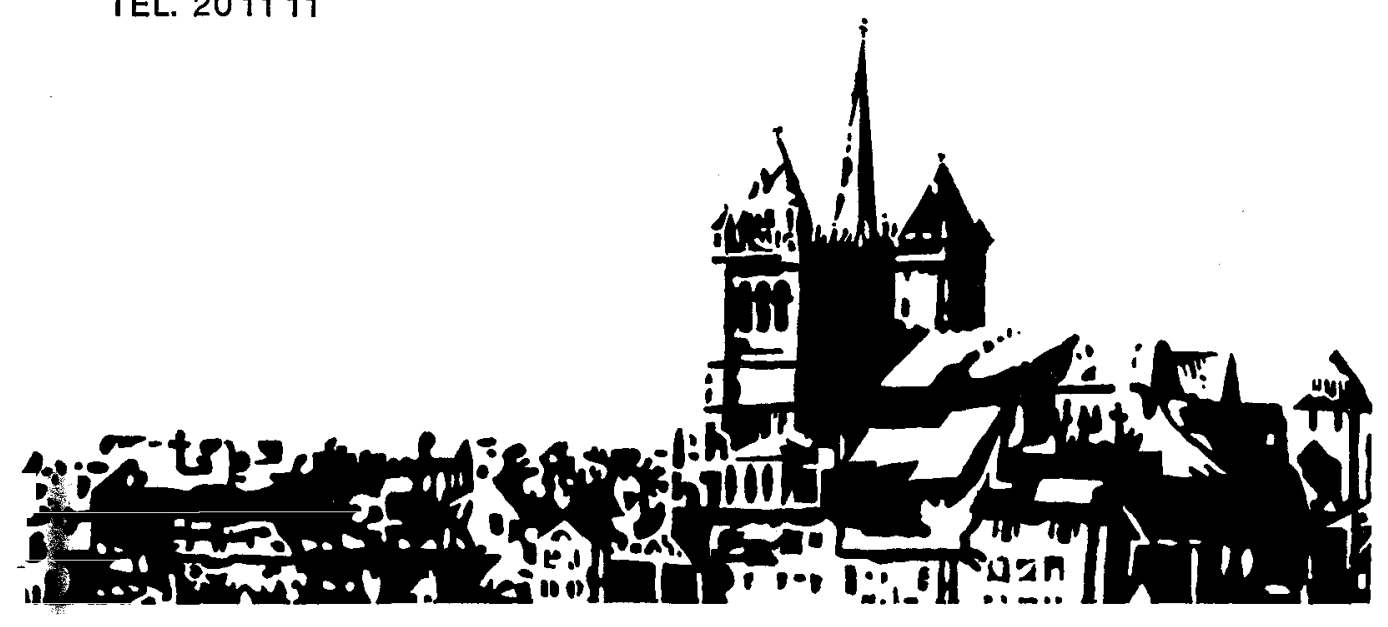




\section{SOCIETE FIDUCIAIRE ROMANDE OFOR S.A.}

Place Saint-Gervais 1, Genève - Tél. 317050

\ Expertises, revisions et organisations comptables

- Domiciliation et administration de sociétés

- Assainissements et liquidations

- Interventions et conseils en matière fiscale

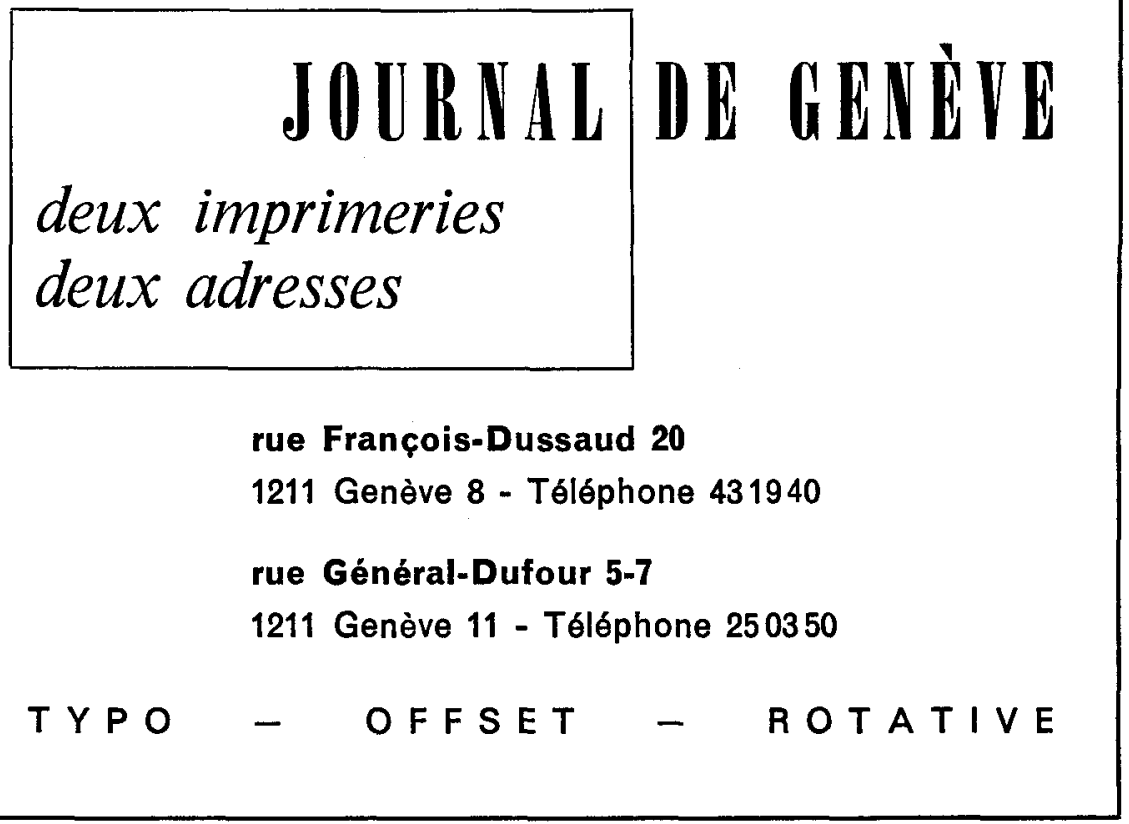




\section{REVUE INTERNATIONALE DE LA CROIX-ROUGE}

SOMMAIRE

COMITE INTERNATIONAL

DE LA CROIX-ROUGE

DANS LE MONDE DE LA CROIX-ROUGE

FAITS ET DOCUMENTS
AOOT $1974-N^{\circ} 668$

Pierre Boissier : Henry Dunant . . . . . . . . . 443

Jean Pictet: Les Conventions de Genève de 1949 ont vingt-cinq ans

L'action du CICR à Chypre . . . . . . . . . 469

Activités extérieures:

Afrique-Amérique latine-Asie-Moyen-Orient . . 475

$A$ Genève :

Décès de $\mathrm{M}$. Adolf Vischer, membre honoraire du CICR ... . . . . . . . . . . . . . . 481

Témoignage de gratitude . . . . . . . . . . . 482

Un film du CICR sur les rapatriements . . . . 483

En Afrique, une fillette rapatriée sous les auspices du CICR . . . . . . . . . . . . . . 484

Belgique . . . . . . . . . . . . . 486

Suisse . . . . . . . . . . . . 487

Collaboration entre la Force d'urgence des Nations Unies et le CICR . . . . . . . . . 488 


\section{INTERNATIONAL \\ REVIEW \\ OF THE RED CROSS}

\section{SUPPLÉMENTS \\ DE LA REVUE}

EN LANGUE ESPAGNOLE

EN LANGUE ALLEMANDE

REVUE INTERNATIONALE DE LA CROIX-ROUGE
Une édition en langue anglaise paraît chaque mois. Elle est en principe identique à l'édition française, et peut être obtenue aux mêmes conditions.

Pierre Boissier: Henry Dunant (I) - Los Convenios de Ginebra de 1949 cumplen veinticinco años Película del CICR sobre repatriaciones - Intervención del CICR en Chipre

Pierre Boissier: Henry Dunant (I) - Die Genfer Abkommen von 194925 Jahre alt - Das IKRK schreitet auf Zypern ein.

Elle est publiée chaque mois par le Comité international de la Croix-Rouge.

17, avenue de la Paix, 1211 Genève (Suisse) - Compte de chèques postaux $12-1767$.

Abonnement un an: Fr. 30,--; le numéro: Fr. 3,-.

RÉDACTION : J.-G. LOSSIER

Seuls les textes signés par le Comité international de la Croix-Rouge engagent la responsabilité de celui-ci. 